\title{
Ser mujer y aprender matemática en tiempo de covid
}

\section{Being a woman and learn mathematics in time of covid}

\author{
Helen Rocío Olivares Chávez*I \\ *Departamento Académico de Educación, Facultad de Educación, Universidad Nacional del Centro del Perú (UNCP), Huancayo-Perú.
}

\begin{abstract}
Resumen
El presente artículo está fundado en el método cualitativa fenomenológico feminista educativo, que comprende las vivencias femeninas reales y sentidas por las mujeres, al observar desde abajo en el aprendizaje matemático femenino en tiempo del COVID - 19. Para mitigar la desigualdad entre hombres y mujeres en el aprendizaje matemático, necesitamos utilizar los procedimientos para resolver problemas matemáticos en las dificultades cotidianas femeninas con el propósito de pensar de forma lógica, se utilizó la técnica de la entrevista semiestructurada, dándole una solución adecuada y oportuna, expandiendo estos procedimientos resolutivos en la sociedad.
\end{abstract}

Palabras Claves: Aprendizaje, matemática, mujer, resolución de problemas, COVID - 19.

\begin{abstract}
This article is based on the educational feminist phenomenological qualitative method, which includes the real feminine experiences felt by women, when observing from below in the feminine mathematical learning in the time of COVID-19. To mitigate the inequality between men and women in mathematical learning, we need to use the procedures to solve mathematical problems in everyday feminine difficulties in order to think logically, the semi-structured interview technique was used, giving it an adequate and timely solution, expanding these resolution procedures in society.
\end{abstract}

Keywords: Learning, mathematics, woman, problem solving, COVID - 19.

1 Correspondiencia: Helen Rocío Olivares Chávez, misshelen007@hotmail.com 


\section{Ser mulher e aprender matemática em tempo de covid}

\section{Resumo}

O presente artigo está fundado no método qualitativo fenomenológico feminista educativo, que compreende as vivencias femininas reais e sentidas pelas mulheres, ao observar desde abaixo no aprendizado matemático feminino em tempo de COVID - 19. Para mitigar a desigualdade entre homens e mulheres no aprendizado matemático, necessitamos utilizar os procedimentos para resolver problemas matemáticos nas dificuldades cotidianas femininas com o propósito de pensar de forma lógica, se utilizou a técnica da entrevista semiestruturada, dando uma solução adequada e oportuna, expandindo.

Palavras Claves: Aprendizado, Matemática, Mulher, Resolução de Problemas, COVID - 19.

\section{Warmi kay yachay matematica kay covid punchao}

\section{Uchuycha}

Kay artículo nisqanruwasqa kan chay método cualitativo fenomenológico warmikunapa alliyachachikuq, kaypin kan warminukunapaqkawsaychan, qawanoraymantayachaymatematicawarmipakaypunchao COVID - 19 Mana chullakakanampaqwarmiwanqariwan matemático yachaypi, Ministaychanichiysasaykunatapaskaypamatematicatapaskayta sapapunchauwarmikasqayraykuallintachayayninmansimiytachachichiy, ruwaspa tecnicataallinrimanakuypi, ruwaspaallintapaskakuyta, Chayachispallapallanayllukunaman.

Hatun apup simi: Yachay, Matematicam, Warmi, Sasaykunata allichay, COVID 19. 


\section{Introducción}

El aprendizaje matemático en las mujeres ha sido, es y será muy importante, porque es una forma de vida. Donde a través del pensamiento lógico se logra alcanzar un conocimiento profundo y exacto de la realidad. Así tomar decisiones acertadas y oportunas para el bienestar. Ruiz (2011), afirma el objetivo de la enseñanza matemática que no es un aprender tradicional, sino la principal finalidad es resolver problemas cotidianos y aplicar conceptos. La fenomenología en educación, no es simplemente un modelo de estudio pedagógico, sino es una forma reflexiva en un sentido profundo. Si es vivido y sentido por una mujer en el ámbito educativo, entonces hablamos del feminismo educativo. Si relacionamos en este tiempo de pandemia, la diferencia de oportunidades entre mujeres y varones, suele continuar en la desigualdad para las mujeres porque se puede generar violencia doméstica.

González (2000), afirma que el profesor es el que reafirma las diferencias de enseñanza por sexo. Considerando que los intereses son diferentes, que conlleva a tratos y aprendizajes diferenciados. La mayoría de mujeres ubicar en primer lugar el papel familiar, ocupaciones domésticas, repartidos con lo profesional, aumentando estereotipos como la matemática es difícil, frente a esta desigualdad surge la necesidad del pensamiento lógico.

Debemos de continuar en pie de lucha, si es necesario con nuestros pensamientos machistas, buscando nuestra esencia como mujeres, aferrándonos al razonamiento. Buscando mejores oportunidades de vida, donde no tenga lugar al resentimiento y frustración de no poder lograr por "ser mujer", concientizándonos de la gran responsabilidad de ser mujeres individualmente y en la sociedad, para lograr un mundo equilibrado.

\section{Feminismo en el aprendizaje matemático}

Es un método de investigación cualitativa fenomenológica educativa. Interpreta la socioconstrucción del aprendizaje matemático femenino desde la parcialidad consciente; visión desde abajo; participación activa en acciones, movimientos y luchas; cambio de statu quo; concientización; historia individual y social; colectivizar sus propias experiencias. Favorece la comprensión y aplicación de cantidad, regularidad, equivalencia, cambio, de forma, movimiento, localización, gestión de datos e incertidumbre en una sociedad en desigualdad de género.

\section{Aprendizaje matemático femenino}

Es el desarrollo de las competencias matemáticas. Dentro del enfoque especifico del área, centrado en resolución de problemas, basado en la lectura y relectura para entender y comprender, identificar los datos, búsqueda de estrategias, aplicación y evaluación respectiva. Con el fin de tomar decisiones matemáticas femeninas oportunas y adecuadas en los problemas cotidianos que se presentan a lo largo de la vida, así lograr la felicidad.

\section{La odisea de la mujer en busca de la igualdad de género}

Nace con la intención de increpar la injusticia social a la que las mujeres que se ven doblegadas a un mundo sometido por el patriarcado, no solo en el Perú sino en el mundo. Herrero (2010) idealizando leyes dirigidas a la igualdad de oportunidad entre hombres y mujeres. Impulsadas por las ideas de la primera obra titulada "Sobre la igualdad de los sexos", escrita a finales del siglo XVII por el francés Paulain de la Barre quien fue el precursor del feminismo. (p. 52)

Suarez (2008), afirma que Adolfo Posada fue un jurista que hizo el primer intento por definir cómo debía ser entendido el feminismo: "movimiento favorable a la mejora de la condición política, social, pedagógica y muy especialmente económica de la mujer" (p. 10), según Martínez (2004) cita a María Mies quien proporciona una línea metodología femenina que tiene procedimientos, sistematizando en siete guías, se contextualizara con hechos trascedentes de lucha: 
Parcialidad consciente: En la revolución francesa, las mujeres llevaron a cabo su propia revolución, sin lograr objetivos, en 1789 se publica en Francia la Declaración de los derechos del hombre, pero no de las mujeres, no estaban dispuestas a darse por sometidas, creando clubes, forma de ejercer la libertad y estar en el ámbito público. Destacando dos obras y dos mujeres, siendo una de ellas Olimpia de Gouges (1748 - 1793) obra “La declaración de los derechos de la mujer y la ciudadanía", muere en la guillotina acusada de feminista.

Una visión desde abajo: Mary Wollstonecraft, la segunda mujer nació en Inglaterra en 1759, escribió "Vindicación de los derechos de la mujer", sometida a la violencia verbal y física del padre, también escribió "Pensamientos acerca de la educación de las niñas", donde avizoraba ideas feministas. Tras la Revolución se obtuvo el nuevo código civil napoleónico plasmando la "ley natural" donde las mujeres quedan declarada menor de edad y a disposición de sus padres, tutores o maridos, sin capacidad económica ni jurídica, ni la patria potestad sobre sus hijos.

Participación activa en las acciones, movimientos y luchas: La lucha de la mujer fue profunda por la igualdad, teniendo como objetivo primordial conseguir el voto y acceder a la educación superior, apareciendo en el siglo XIX el feminismo como movimiento social internacional, viviendo humillaciones y traiciones por partidos Republicanos entre otros, donde este movimiento se unieron para radicalizar su lucha, consiguiendo aprobar la ley de sufragio las inglesas en 1917, también las estadounidenses lograron en 1918, siendo consecuencia a la adquisición de la educación de la mujer.

El feminismo en Perú tiene su historia de lucha, Silva (1984), señala la Colla nunca pudo aspirar a ser gobernante, ni Sumo Sacerdote, ni Amauta, ni Quipucamalloc (estadista del imperio), solo realizaría trabajos domésticos. Con la invasión española se estableció el feudalismo estableciendo una sociedad marcada en la jerarquización, con mayor división de clases explotadoras, que aprovechaba la mano de obra india, la mujer fue la principal víctima de subordinación, discriminación, opresión y degradación sexual.

Considerada la primera feminista peruana María Jesús Alvarado (1878 - 1971), nació en Chincha Baja, estudio hasta tercer grado de primaria, máximo grado que podían estudiar las mujeres, donde la exigencia era fuerte que terminaban leyendo y escribiendo, siendo la más sobresaliente de la clase, clausurando con un discurso que dio inicio a luchar por los derechos de las mujeres, sus estudios posteriores fueron autodidactas, apoyada de un primo que era catedrático de la UNMSM consigue empleo como columnista en el diario El Comercio.

También desarrollo conferencias "El Feminismo en la Sociedad Geográfica de Lima". Exponiendo los fundamentos femeninos como corriente de cambio social a nivel mundial, igualdad de derechos civiles y políticos de las mujeres. Fundo la institución en pro de la cultura y los derechos de la mujer llamado "Evolución femenina", que tenía el propósito de crear un colegio secundario público para mujeres, aprobándose por la Cámara de Diputados y luego en la Cámara de senadores del Perú. Sin embargo, otras naciones lograban el derecho del voto y la educación superior, el Perú en 1936 solo se lograba que las mujeres casadas dejaron la custodia del esposo, podían administrar sus bienes, testiguar, contratar, entre otros puntos similares. Pero en 1955 el gobierno del presidente Manuel A. Odría, promulga la Ley 12391 que permite a la mujer toda la amplitud de sus derechos ciudadanos al igual que el hombre. El Perú fue el penúltimo país de América Latina en el voto femenino.

Cambio del status quo: El movimiento se expandió internacionalmente, con la Declaración Universal de los Derechos Humanos, se continuó luchando por la igualdad en todos los estamentos reclamando a la genuina universalización de los valores democráticos, donde las mujeres obtendrían oportunidades laborales con el mismo salario que el hombre, administrar sus bienes, la igualdad en el matrimonio de compartir la potestad de los hijos. Ingresado el capitalismo y dividiendo en dos perfiles, por una parte, las mujeres proletarias y la otra: burguesas.

Así, tenemos a una de las defensoras de los derechos de las mujeres en la educación Sofía Kovalevskaya, nacida en Moscú en 1850, que desde muy niña tuvo la habilidad de resolver problemas matemáticos, siendo la primera mujer que logro una plaza de profesora universitaria en Suecia Europa, teniendo muchos obstáculos solo por ser mujer. Aportando ecuaciones diferenciales, anillos de Saturno y programación de la luz, consiguió el premio Bordin, con el trabajo sobre el problema de la rotación de un cuerpo alrededor de un punto fijo. 
Concientización: El filósofo francés Simon Beauvoir en su libro "El segundo sexo", escribió en 1949, analizando a la mujer como el otro lado del espejo de la evolución del mundo masculino, motivando la lucha femenina a reconquistar su propia identidad, ya que ha sido la otra respecto del varón. El sufragio no hizo que el papel de la mujer cambie, afirma la Norteamericana Betty Friedan en su libro "La mística de la feminidad", experimentando una sensación de vacío, esforzándose en una vida profesional y familiar.

Historia individual y social: Elizabeth Garrett Anderson, estudio por su cuenta, porque se le negó el ingreso a las escuelas de medicina por ser mujer, convirtiéndose en 1865 la primera doctora británica, creando una facultad médica para mujeres, luego la clínica tomo su nombre quedando renombrado como el Nuevo Hospital para mujeres, contribuyo exigiendo el voto femenino y la educación superior.

Colectivizar sus propias experiencias: María Enríquez Ladrón de Guevara (1846 - 1891), primera mujer Cuzqueña peruana y de Sudamérica que estudio Derecho, fundó el colegio superior para mujeres, elogiados por algunos y agredidas por otros, cerrando después de tres años, superando estos inconvenientes en 1874 el gobierno emitió una resolución suprema autorizando rendir examen en cualquier universidad. Ingresando a la Universidad San Antonio Abad del Cusco 1875, durando la evaluación diez días frete a un riguroso jurado, obteniendo una alta calificación.

En 1878 se graduó como bachiller en jurisprudencia, no como abogada, porque en esa época estaba prohibido entregar títulos profesionales a las mujeres, demandando su caso al Poder Judicial y el congreso, no tuvo éxitos, pero motivó e inspiró a otras mujeres para continuar sus pasos, el presidente Nicolás de Piérola le sede la posibilidad de recibirse como abogada, pero no aceptó solidarizándose con las demás mujeres que estaban impedidas de estudiar. En 1870 logró abrir una escuela nocturna para obreros, enseñándoles a leer, escribir y conocer leyes.

\section{Participación de las mujeres en matemática}

Muchas mujeres matemáticas fueron ignoradas, no reconocidas y olvidadas. Tenemos a Elisa de Tiro, más conocida como Dido, huye del hermano que había matado a su esposo a las costas de África, pidiendo hospitalidad y tierras. El Jarbas le dijo que le daría tanta tierra como ella pudiera abarcar con una piel de buey, abarcando lo máximo posible corto en finas tiras, consiguiendo circunscribir un extenso perímetro, originando este problema matemático. En la comunidad Pitagórica, las mujeres participaron de forma secreta, como Theano discípula de Pitágoras y luego esposa, dirigió y enseñó en la escuela, se le atribuye escritos de matemática, física, medicina, psicología infantil, tras una rebelión en la escuela se infiere que Pitágoras muere y Theano huyó con su hija, difundiendo las ideas Pitagóricas. Grecia era una ciudad patriarcal, pero en Esparta las mujeres tuvieron un poco de poder.

En el siglo V a. C, tenemos a Perictione, madres de Platón que escribió sobre obligaciones de las mujeres, enseñando a muchas de ellas matemática, como a Diótimia, Sócrates hablaba que ella, que era una maestra sacerdotisa, además Aglaonice de Tesalia famosa por predecir eclipses utilizando conocimientos matemáticos, fue considerada una hechicera. Debido a las leyes del hombre que suprimían a las mujeres de reuniones públicas, algunas eran extranjeras o se disfrazaban de hombres para asistir a estos eventos académicos. También tenemos a Hipita de Alejandría matemática nacida en el siglo III, trabajó en comentarios de la Aritmética de Diofanto y las Secciones Cónicas de Apolonio. Asimismo, tenemos a Sophie Germain matemática autodidacta francesa (1776), utilizó el seudónimo Monsieur LeBlanc para que le tomen en serio por el hecho de ser mujer, aportando la teoría de números, geometría, introdujo el concepto de curvatura media de una superficie.

En 1919, nació Julia Robinson matemática estadounidense, fue la primera mujer miembro de la Academia Nacional de Estados Unidos y presidenta de la American Mathematical Society, aportando en ecuaciones diofánticas. Además, en 1900 nace la matemática inglesa Mary Cartwright, se doctoró, aportando en el campo de las ecuaciones diferenciales esenciales en el desarrollo de la radio y el radar, creando el teorema de Cartwright sobre máximo de funciones, siendo la primera mujer en conseguir la medalla Sylvester y ser presidenta de la London Mathematical Society. 
En 1918, nació la matemática afroamericana Katherine Johnson, contratada por la NASA, su exactitud en los cálculos le admitió calcular la trayectoria del viaje del Apolo 11 a la Luna en 1969, también tenemos a Maryam Mirzakhani matemática iraní, primera mujer en lograr una medalla Fields (2014), una de los mayores honores de un matemático, por colaborar al estudio de los espacios de moduli de la superficie de Riemann, así tenemos muchas mujeres que han contribuido con la evolución en la matemática, para solucionar problemas diversos del mundo.

\section{La entrevista}

Se entrevistó a la estudiante de iniciales KSDE (12 años), que cursa el primer grado de secundaria, en una institución educativa pública, natural de Huaricolca, ella es la sexta hermana de 8 , siendo cinco mujeres y tres varones (hermanos mayores), su padre trabaja actualmente como controlador del ingreso de personas y vehículos al distrito de Huaricolca, desinfectando para no contagiar a la comunidad del COVID - 19, el padre culmino la secundaria.

Su madre se dedica al pastoreo en la estancia, viviendo con su hermana que le antecede y sus dos hermanos menores (mujer 9 años y varón 7 años), saliendo una vez a la semana del lugar, ella se quedó en segundo grado de secundaria porque se embarazó de su hermano mayor, Huaricolca es un distrito considerado de extrema pobreza solo cuando llueve se siembra, otras fechas es un poco triste, ya que no hay movimiento económico.

\section{Diálogo de la entrevista}

Respecto a la primera pregunta, ¿Quién crees que aprende mejor la matemática: el varón o las mujeres?, la segunda ¿quién te gustaría que sea tu profesor de matemática, un varón o una mujer? y tercera igeneralmente, en tu casa quién o quiénes realizan las actividades del hogar?, de parcialidad consciente: La estudiante responde que la mujer aprende y enseña mejor la matemática. Cuando participa activamente en clases. Resolviendo problemas matemáticos y obteniendo mejores calificativos.

Pregunta cuatro. ¿Extrañas que las clases de matemática sean de forma presencial? ¿Por qué? y la pregunta cinco ¿por qué medio te comunicas con tus profesores en este tiempo de pandemia? corresponden a una visión desde abajo, respondiendo que extraña las clases presenciales, porque puede jugar con sus amigas, utiliza los materiales ambientes que proporciona la institución educativa. Ya que ella se presta el celular de su hermano para realizar las clases grupales desarrollado por el whatsApp, otras fechas no puede ingresar a clases grupales ni individuales porque no cuenta con el celular.

Pregunta seis. Las actividades que tú realizabas en tu hogar, ¿son las mismas que realizas en esta pandemia?, atañe a la participación activa en acciones, movimientos y luchas, respondiendo la estudiante que no realiza las mismas actividades antes de la pandemia, ahora se incrementaron sus acciones como realizar el almuerzo y cena, atender al padre que viene de trabajar, a sus hermanos y hermanas cuando vienen de cosechar maca e ingresar a clases cuando me deja su celular.

Pregunta siete. Por el hecho de ser mujer ¿crees que tienes mayor o menos oportunidades de continuar estudiando? y pregunta ocho ¿crees que resolver problemas matemáticos te ayudarán a resolver tus propios problemas o dificultades que te puedan ocurrir en la vida? ¿Cómo?, direccionado al cambio de statu quo, responde la estudiante, tengo mayor oportunidad de salir adelante, mi mamá no pudo estudiar, pero ella me dice que estudie, para tener mis propias cosas, resolver problemas matemáticos si me ayudan a resolver mis problemas, pensando mejor.

Pregunta nueve. ¿qué harías como alcaldesa del Municipio Escolar de tu colegio?, corresponde a concientización, la estudiante manifiesta que realizaría una lista para organizar la utilización de la cancha deportiva también por las mujeres, podría ser un día que jueguen los varones, un día las mujeres, poner disciplina en el recreo y cambio de clase porque hay alumnos que a veces se pelean y nos molestan, así nos sentiremos mejor en el colegio. 
Pregunta diez, ¿podrías describir los pasos para resolver un problema matemático?, once ¿los pasos para resolver un problema matemático, se podrá utilizar para resolver un problema cotidiano? y pregunta doce ¿conoces alguna mujer, que utilice los conocimientos matemáticos, para realizar su trabajo?, corresponde a la historia individual y social, respondiendo la entrevistada que sí, describiendo que primero debemos entender el problema, de allí debemos identificar los datos.

Luego entender si utilizaremos suma, resta, multiplicación o división y sacando eso nos sale la respuesta, pero de ahí debemos identificar si está bien o está mal, si está mal vuelves a intentarlo. Si, los pasos nos pueden ayudar a resolver nuestros problemas que nos sucedan, razonando, pensar de qué problema está pasando, identificando bien. Si conozco la señora Carmen es contadora, trabaja en un banco, también la señora Nora escoge su papa, primera, segunda y tercera para vender.

La pregunta trece. Estos pasos de resolver problemas matemáticos ¿liberará a las mujeres oprimidas y buscarán oportunidades?, esta pregunta corresponde a colectivizar sus propias experiencias de la mujer, respondiendo la entrevistada si, nos ayuda bastante a las mujeres, porque aquí hay mujeres maltratadas, que discuten por el dinero, se gritan cuando no tiene lo suficiente, se insultan, debemos de pensar y razonar bien para que esto no nos pase.

Mi profesora de la primaria siempre nos decía que estudiemos mucho las mujeres para que no las maltraten los hombres abusivos y mi madre siempre habla que aprovechemos el estudio para no sufrir como ella, que está pastando animales de otras personas, para comprar lo que necesitamos en el colegio, que no hagamos caso a mi papá y hermanos cuando nos dicen para atender al esposo no necesitan tanto estudiar, es que mis hermanas terminaron la secundaria, pero ninguna continuo estudiando, yo sí quiero.

\section{Conclusiones}

La entrevistada tiene la actitud y pre disponibilidad de aprender, pese a las condiciones hostiles que vive, en el aspecto económico y familiar, pero ella utiliza los pasos para resolver problemas matemáticos, relacionando estos pasos para resolver cualquier problema que se le presente en la vida cotidiana, entonces es una alternativa para las mujeres de tomar decisiones más acertadas y oportunas en nuestras vidas, utilizando el razonamiento. 


\section{Referencias Bibliográficas}

González, J. (2000). Políticas públicas en material de género y educación: análisis del caso mexicano. En: Construyendo la diversidad de nuevas orientaciones en género y educación. México. Porrúa.

Herrero, R. (2010). La imagen de la mujer en la prensa entre 1910 -1915 y 2000 - 2005: Estudio comparado. Tesis Doctoral. Recuperado de https://eprints.ucm.es/11025/1/T32151.pdf. Universidad Complutense de Madrid. Madrid.

Martínez, M. (2004). Ciencia y Arte en la Metodología Cualitativa. Recuperado de https://www.academia. edu/29811850/Ciencia_y_Arte_en_La_Metodologia_Cualitativa_Martin ez_Miguelez_PDF. México: Trillas.

Ruiz, Y. (2011). Aprendizaje de la matemática. Revista digital para profesionales de la educación. Federación de enseñanza. Andalucía - España.

Silva, E. (1984). Situación laboral, legal y política de la mujer peruana. Presentado al VII Congreso Nacional de Enfermería. Lima.

Suarez, C. (2008). Historia del mundo feminista. Oviedo. Instituto Asturiano de administración pública "Adolfo Posada". 